\title{
Stress relief via island formation of an isotropically strained bimetallic surface layer: The mesoscopic morphology of the Ag/Pt (111) surface alloy
}

\author{
R. Schuster, ${ }^{*}$ H. Röder, K. Bromann, H. Brune, and K. Kern \\ Institut de Physique Expérimentale, Ecole Polytechnique Fédérale de Lausanne, Switzerland
}

(Received 3 June 1996)

\begin{abstract}
Upon annealing above $620 \mathrm{~K}$, submonolayers of Ag deposited on $\operatorname{Pt}(111)$ are known to mix into the first surface layer. Thereby the individual $\mathrm{Ag}$ atoms reduce the strain fields caused by the lattice mismatch. As observed by scanning tunneling microscopy, careful annealing of the intermixed surface leads to the formation of small round islands with a preferential diameter of $150-300 \AA$ on terraces wider than a critical width of about $400 \AA$. This indicates that the microscopically intermixed surface is still under considerable stress, which is released by a mesoscopic change of the morphology. An effective release of elastic stress at the island and step edges is thought to compensate for the formation energy of steps and the repulsive elastic step-step interaction. [S0163-1829(96)03840-4]
\end{abstract}

\section{INTRODUCTION}

Elastic strain, caused by crystalline mismatch, is well known to have a profound influence on the morphology of a coherent two-phase alloy. A precipitate-matrix misfit in such systems can drive a nearly random spatial phase distribution into a highly ordered domain structure. ${ }^{1}$ The changes in morphology and spatial correlation occur on a mesoscopic length scale, due to the long-range nature of the strain-induced elastic interactions between precipitates.

In recent years it has been realized that surface stress can have very similar effects on the surface morphology. ${ }^{2,3}$ For example, the intrinsic tensile stress of the $\mathrm{Au}(111)$ surface, caused by the reduced number of nearest neighbors of the surface atoms, drives this surface into a herringbone reconstruction, with a mesoscopic domain width of $\approx 150 \AA \AA^{4,5}$ Similarly, externally imposed stress may lead to the same phenomena: Upon $\mathrm{O}$ adsorption on $\mathrm{Cu}(110)$ there form striped arrays of the $(2 \times 1) \mathrm{O} / \mathrm{Cu}(110)$ structure spontaneously on the clean $\mathrm{Cu}$ surface. ${ }^{6}$ Whereas in these examples the surface structure constitutes a patchwork of domains with either anisotropic or even intrinsically different stress tensors in the different domains, similar effects have also been observed at surfaces which do not form different surface domains. For example, on $\operatorname{Pd}(110)$ a regular arrangement of islands was found even though no obvious difference between the surface on the islands and the terrace exists. ${ }^{7}$

Marchenko ${ }^{8}$ and Alerhand et al. ${ }^{9}$ pointed out that in the case of anisotropic surface domains, i.e., different stress tensors in the different domains (far away from the steps), the elastic stress can be released very effectively by the formation of domain boundaries. Domain boundaries here refers to boundaries between any kind of surfaces patches, as there are, for example, steps or walls between different domains. The elastic relaxation energy diverges logarithmically with the distance between the domain boundaries and hence eventually compensates for the energy necessary for the formation of an isolated domain boundary. This model, however, is not applicable when no difference of the stress tensor between neighboring domains exists, as is the case with
$\operatorname{Pd}(110) .^{7}$ Here the elastic repulsion of the steps is proportional to the inverse of their squared distance. ${ }^{10}$ As this repulsion results in a positive contribution to the surface free energy for all step distances, the authors introduced a negative formation energy for an isolated step to stabilize such regular arrangements of islands. Assuming an effective local relaxation of surface stress at step edges, this seems very reasonable but at the same time opens new questions, e.g., how roughening of the surface is prevented.

In this paper, we present scanning tunneling microscopy (STM) results on the formation of islands on an isotropically stressed surface, the surface alloy of $\mathrm{Ag} / \mathrm{Pt}$. Depositing $\mathrm{Ag}$ on the $\mathrm{Pt}(111)$ surface at coverages below one monolayer and slight annealing to temperatures above $620 \mathrm{~K}$ results in the formation of a surface alloy: $\mathrm{Pt}$ and $\mathrm{Ag}$ intermix in the first layer and form a uniform surface layer, consisting of small Ag clusters $(\approx 10 \AA)$ embedded in the Pt surface. ${ }^{11}$ The intermixing is restricted to the first layer. As pointed out by Tersoff, the simple framework of surface and interface energies of the two respective species is not sufficient to explain the observed results, i.e., the miscibility at the surface with the bulk solubility tending towards zero. ${ }^{12}$ What is neglected in this simple picture is the difference between the stress due to the lattice misfit of a simple $\mathrm{Ag}$ monolayer and that of the intermixed layer. Indeed, Tersoff proved that there occurs mixing in the surface layer despite a positive interface energy between the two species: The strain fields of the deposited atoms tend to separate them and mix them into the surface layer. Intermixing into deeper layers is strongly unfavorable. The positive interface energy between the two species leads to clustering, as observed in the experiment.

Due to the lattice mismatch of $\mathrm{Ag}$ and $\mathrm{Pt}$ (nearestneighbor distances: $d_{\mathrm{Ag}}=2.89 \AA$; $d_{\mathrm{Pt}}=2.77 \AA$ ), this uniform surface mixture is expected to be under considerable stress, which might render this surface unstable against relaxation, at least on large length scales. In this paper, we show that careful annealing of the (already microscopically intermixed) surface leads to the formation of islands on the larger terraces. We followed the process of their formation by STM: In the course of the annealing large islands break apart into smaller ones. This rather stable arrangement of round islands 
shows a peaked width distribution with an average island diameter of 150-300 $\AA$, slightly depending on the terrace width, which indicates an interaction between island and terrace steps. As there is no difference of the surface structure in the islands and on the terraces, the main elastic interactions of the steps are supposed to follow the above $1 / l^{2}$ law, where $l$ is the distance between the steps. Compensation of that step repulsion and their formation energy has to be sought in a very efficient relief of strain perhaps due to lateral relaxation of the islands.

\section{EXPERIMENTAL RESULTS}

The experiments were performed with a variable temperature UHV scanning tunneling microscope as described in Ref. 13. The Pt(111) surface was prepared by subsequent cycles of Ar sputtering, annealing in oxygen atmosphere, and flash annealing to $1200 \mathrm{~K}$. Ag was evaporated from a Knudsen cell with a boron nitride crucible at a rate of $1 \mathrm{ML}$ per $900 \mathrm{~s}$. After annealing of the sample to the respective temperatures, the STM images were recorded at $400 \mathrm{~K}$.

Figure 1(a) shows the Pt surface after deposition of $0.55-\mathrm{ML} \mathrm{Ag}$ at $400 \mathrm{~K}$ and annealing to $550 \mathrm{~K}$ for $100 \mathrm{~s}$. The $\mathrm{Ag}$ forms triangular islands on the terraces. From previous work the $\mathrm{Ag}$ is known to occupy fcc sites. ${ }^{14}$ The straight island edges are running along the close-packed [110] directions. The preference of B-type steps, ${ }^{15}$ forming (111) microfacets leads to the triangular appearance of the islands in Fig. 1(a). The Pt steps are wetted by Ag. Additionally a few white spots are resolved, probably representing mobile and metastable Ag clusters as discussed in Ref. 16. Besides Fig. 1(b), all STM images shown in this paper are high pass filtered and hence appear as illuminated from the left-hand side. The colors in these images do not correspond to absolute heights, rather to relative heights of neighboring patches, where the brighter color corresponds to ascending of the STM tip. The feature in Fig. 1(a), marked with $A$ corresponds to a $\mathrm{Ag}$ seamed ascending step edge (upon going from left to right), whereas $B$ marks a $\mathrm{Ag}$ island on the Pt surface. These identifications of the features were made in more detail in Ref. 11. In STM images (not shown), depicting the absolute heights of the tip at constant tunneling current, the $\mathrm{Ag}$ is imaged approximately $0.6 \AA$ higher than the Pt. This holds true for all applied tunneling conditions $(-0.5 \mathrm{~V}$ $\leqslant U_{T} \leqslant-1.5 \mathrm{~V} ; 1 \mathrm{nA} \leqslant I_{T} \leqslant 3 \mathrm{nA}$ ), and for the intermixed phase. As the true difference of the atomic radii amounts only to $0.1 \AA$, this cannot be a purely geometric effect. Differences in the electronic structure, due to strain within the Ag patches, might be responsible for the observed imaging heights. ${ }^{11,17}$

In Fig. 1(b) after $100 \mathrm{~s}$ of annealing at $630 \mathrm{~K}$ the intermixing of $\mathrm{Ag}$ and $\mathrm{Pt}$ starts, most obvious from the changed island morphology, where the triangular islands have transformed into rather round ones. On a microscopic scale small amounts of (dark) Pt indentations of $\approx 10 \AA$ size have formed in the Ag islands. On the other hand, the Pt terraces reveal (bright) Ag protrusions preferentially at the Ag wetted Pt step edges. As discussed in detail in Ref. 11, the intermixing process occurs very effectively at the step edges, where only intralayer mass transport is required. But also some interlayer mass transport is kinetically allowed as follows from
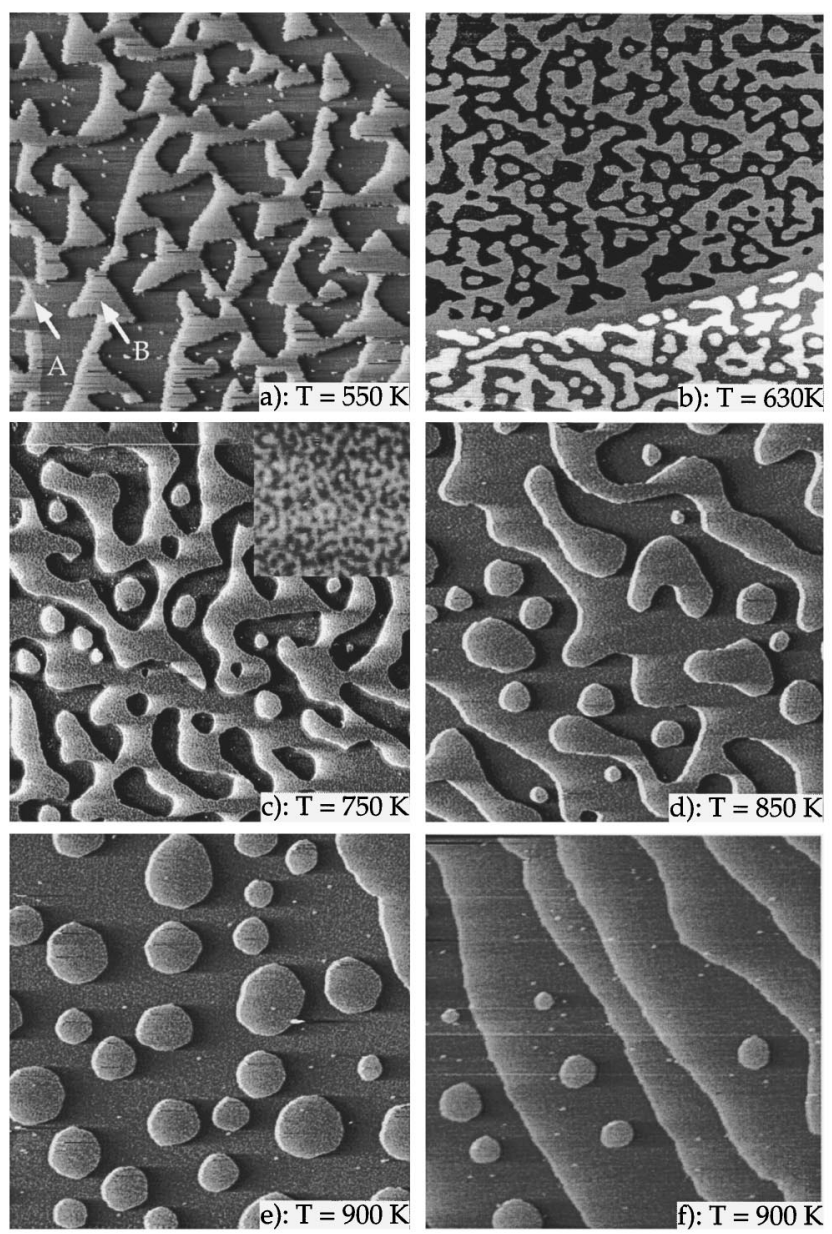

FIG. 1. STM topographs of the $\operatorname{Ag} / \mathrm{Pt}(111)$ system with $\theta_{\mathrm{Ag}}=0.55 \mathrm{ML}$ after annealing to different temperatures. The STM images were taken at $400 \mathrm{~K}$; (a) annealing at $550 \mathrm{~K}$ for $100 \mathrm{~s}$ formed triangular-shaped $\mathrm{Ag}$ islands on the terraces; $A$ marks an Ag-seamed upward step, $B$ marks a $\mathrm{Ag}$ island on a Pt terrace. (b) additional annealing at $630 \mathrm{~K}$ for $100 \mathrm{~s}$ started the mixing of the $\mathrm{Ag}$ into the Pt surface. (c) After subsequent annealing to $750 \mathrm{~K}$ for another $100 \mathrm{~s}$, the intermixing was completed [the inset $(225 \times 225$ $\AA$ ) reveals the microscopic morphology of the Ag-Pt surface alloy]. (d) Further annealing at $850 \mathrm{~K}$ for $100 \mathrm{~s}$ caused meandering islands of the $\mathrm{Ag} / \mathrm{Pt}$ mixture with fingerlike protrusions. (e) Another annealing for $100 \mathrm{~s}$ at $900 \mathrm{~K}$ leads to the final structure, round intermixed islands on large terraces, which are also homogeneously mixed. (f) These islands exist only on terraces above a critical width of $\approx 400 \AA$. The image sizes are $2300 \times 2300 \AA^{2}$. All images except (b) are high pass filtered (see text)

detailed investigations of the intermixing of isolated Ag islands with the substrate $\mathrm{Pt}$ on large terraces (not shown here). They revealed higher cluster concentrations in the vicinity of the island perimeters. This holds true for both the Ag clusters mixed into the terraces and for the Pt clusters mixed into the $\mathrm{Ag}$ islands and implies that also the interlayer mass transport proceeds preferentially at the edges of the islands.

Additional annealing to $750 \mathrm{~K}$ for $100 \mathrm{~s}$ leads to complete intermixing of $\mathrm{Ag}$ and $\mathrm{Pt}$ in the top layer, as can be seen from the inset in Fig. 1(c). At 0.55-ML Ag the Ag clusters (bright) in the topmost layer touch already and form a network with a typical thickness of $\approx 10 \AA$. It should be noted 
that the intermixing can also be achieved by longer annealing at $630 \mathrm{~K} .{ }^{11}$ Meandering interconnected islands have formed on the terraces, sometimes including holes and round islands. Closer inspection reveals that the islands are surrounded by $\approx 10$ - $\AA$-wide rims, imaged at the same height as the Ag clusters. They are therefore interpreted as small Ag seams, wetting the island and step edges. The preferred distance between the islands is about 50-100 $⿱$. In Fig. 1(c) the islands cover still about $50 \%$ of the terrace, as is expected from the deposited amount of Ag.

Upon subsequent annealing to $850 \mathrm{~K}$ for $100 \mathrm{~s}$ [Fig. 1(d)], the meandering islands develop fingerlike protrusions, with a width of about $200 \AA$. Most of the holes in the meandering islands have disappeared, and the fraction of the surface covered by islands has considerably reduced, indicating effective-mass transport of $\mathrm{Ag}$ and $\mathrm{Pt}$ towards the terrace edges.

Heating to $900 \mathrm{~K}$ for an additional $100 \mathrm{~s}$ leads to the final configuration of the intermixed surface [Fig. 1(e)]. One ends up with fairly round-shaped islands of an average diameter of $275 \AA$ and a fairly narrow size distribution with a standard deviation of $\pm 80 \AA$. The STM images show no difference in the local morphology of the surfaces on islands and terraces. According to the former experimental results ${ }^{11}$ and the theoretical considerations of Tersoff, ${ }^{12}$ the $\mathrm{Ag}$ mixes only into the topmost layer. The island edges are still seamed by a $\approx 10$ - $\AA$-wide rim of $\mathrm{Ag}$. Annealing to higher temperatures does not change the overall morphology, even though at slightly higher temperatures considerable thermal desorption of $\mathrm{Ag}$ sets in. It is also worth mentioning that the same morphology is obtained by annealing half a monolayer of $\mathrm{Ag}$ on $\mathrm{Pt}(111)$ at $630 \mathrm{~K}$ for $100 \mathrm{~min}$. The average width of the islands depends slightly on the width of the terrace on which they are formed, with smaller terraces leading to smaller island sizes. However, the narrow-width distribution with a standard deviation of about a quarter of the average width remains unchanged. In Fig. 1(f) it can be seen that these islands form only on terraces with a critical width above $\approx 400 \AA$.

\section{DISCUSSION}

Why do these small homogeneously distributed islands form at all? There are no topological constraints, which could prevent the meandering islands in Figs. 1(c) and 1(d) from collapsing into large compact islands. Nevertheless, the meandering islands decay into smaller ones. Additionally, the area covered by islands on a terrace reduces considerably during the first steps of the annealing sequence in Fig. 1. On smaller terraces these islands even dissolve completely. This substantial mass transport towards the step edges stops once the island structure has formed, and there is no obvious effect of further annealing. Only after 10-min annealing at 900 K, where already about $20 \%$ of the Ag had desorbed, slight modification of the island density was found. Furthermore a formation of the islands due to condensation of a twodimensional gas of $\mathrm{Ag}$ and $\mathrm{Pt}$ atoms or small clusters upon the quenching from the annealing temperature to the observation temperature of $400 \mathrm{~K}$ can be ruled out: The same island morphology has been obtained with both procedures, stepwise annealing up to $900 \mathrm{~K}$ (for $100 \mathrm{~s}$ ) and annealing at
$630 \mathrm{~K}$ for $100 \mathrm{~min}$. Assuming the equilibrium between the (solid) terraces and a two-dimensional gas phase following the Clausius Clapeyron equation, the density of the twodimensional gas on the terraces should depend exponentially on the temperature. Hence also island density and size were expected to vary significantly between the two annealing procedures. Additionally, STM images recorded directly at $650 \mathrm{~K}$ do not reveal any hints for a mobile, dilute $\mathrm{Ag}-\mathrm{Pt}$ phase on the terraces, which is further supported by the absence of detectable mobility of the steps of the surface alloy, even at $650 \mathrm{~K} .^{18}$

From both of the above arguments, i.e., no evident influence of neither the further annealing nor the annealing procedure, we conclude that these islands constitute the equilibrium structure of this stressed surface layer on terraces above a critical width of $\approx 400 \AA$. We do not want to claim that this morphology necessarily constitutes the absolute thermal equilibrium structure of the surface, which could, e.g., consist of a stepped surface with terrace widths below the observed critical one. Such a rearrangement would require long-range mass transport over several surface layers and might be kinetically suppressed up to the desorption temperature of $\mathrm{Ag}$.

A clue for the understanding of the island structure can be found in their local distribution. They are more or less homogeneously spaced, indicating mutual repulsion. Also, the concave step edge in Fig. 1(f), bending away from the islands on the lower terrace substantiates this implication. A straightforward explanation is long-range step-step repulsion, due to elastic stress in the crystal surface. As mentioned above, such a repulsion between steps, which separate domains with no significant difference in their stress tensor, should decrease with $1 / l^{2}$, where $l$ is the separation between steps. This positive repulsion energy has to be compensated by a negative-energy contribution to stabilize the island pattern. As the fairly round island shape indicates a tendency for the steps to shorten and hence a positive formation energy for an (isolated) step, such a contribution has rather to be sought in the effective relaxation of the islands themselves. Indeed, in recent reflection high-energy electron-diffraction investigations of the homoepitaxy of $\mathrm{Cu}$ on $\mathrm{Cu}(001)$ Fassbender et al. found a periodic oscillation of the in-plane lattice constant with the layer thickness, which was interpreted in terms of lateral relaxation of $\mathrm{Cu}$ islands at incomplete monolayers of $\mathrm{Cu} .{ }^{19}$ Such a relaxation effect, whose efficiency should increase with decreasing diameter of the islands, could overcompensate the step-step repulsion and the formation energy of a step for small island diameters and might therefore lead to the stabilization of the observed surface morphology. It should also be noted that the Ag seams, covering the step edges, signal an enhanced adsorption energy for Ag atoms at these step edges, which is also thought to considerably decrease the formation energy of the steps.

\section{CONCLUSIONS}

We showed that the observed mechanism, the spontaneous formation of islands, is effective for the relief of surface stress even in systems with isotropic surface domains. This is principally different from systems like the herringbone reconstruction on $\mathrm{Au}(111)$ or the striped arrangement of $(2 \times 1)$ 
domains on $\mathrm{O} / \mathrm{Cu}(110)$, where long-range elastic relaxations due to the anisotropy of the stress tensor in the different domains compensate for the elastic repulsion and formation of the domain boundaries. In our case we reported on a system, where the relaxation of the domains themselves, depending on their lateral extent, is considered to compensate for the mutual step-step repulsion and the step formation energy. Such a lateral relaxation might also be the explanation for the observed formation of islands on the $\operatorname{Pd}(110)$ surface. It would then become unnecessary to introduce a negative step formation energy, which could furthermore solve the problem of surface roughening, expected to result from such a negative step formation energy.

\section{ACKNOWLEDGMENTS}

The authors thank S. Narasimhan, E. Pehlke, H. Ibach, and P. Zeppenfeld for fruitful discussions and comments.
*Permanent address: Fritz-Haber-Institut der Max-PlanckGesellschaft, Berlin, Germany.

${ }^{1}$ H. Kubo, I. Cornelis, and C. M. Wayman, Acta Metall. 28, 405 (1980).

${ }^{2}$ D. Vanderbilt, in Computations for the Nano-Scale, edited by P. E. Blöchl (Kluwer, New York, 1993), p. 1.

${ }^{3}$ P. Zeppenfeld, M. Krzyzowski, C. Romainczky, G. Comsa, and M. G. Lagally, Phys. Rev. Lett. 72, 2737 (1994).

${ }^{4}$ J. V. Barth, H. Brune, G. Ertl, and R. J. Behm, Phys. Rev. B 42, 9307 (1990).

${ }^{5}$ S. Narasimhan and D. Vanderbilt, Phys. Rev. Lett. 69, 1564 (1992).

${ }^{6}$ K. Kern, H. Niehus, A. Schatz, P. Zeppenfeld, J. Goerge, and G. Comsa, Phys. Rev. Lett. 67, 855 (1991).

${ }^{7}$ H. Hörnis, J. R. West, E. H. Conrad, and R. Ellialtioglu, Phys. Rev. B 47, 13055 (1993).

${ }^{8}$ V. I. Marchenko, Pis'ma Zh. Éksp. Teor. Fiz. 33, 397 (1981) [JETP Lett. 33, 381 (1981)].

${ }^{9}$ O. L. Alerhand, D. Vanderbilt, R. D. Meade, and J. D. Joannopoulos, Phys. Rev. Lett. 61, 1973 (1988).
${ }^{10}$ V. I. Marchenko and A. Y. Parshin, Zh. Éksp. Teor. Fiz. 79, 257 (1980) [Sov. Phys. JETP 52, 129 (1981)].

${ }^{11}$ H. Röder, R. Schuster, H. Brune, and K. Kern, Phys. Rev. Lett. 71, 2086 (1993).

${ }^{12}$ J. Tersoff, Phys. Rev. Lett. 74, 434 (1995).

${ }^{13}$ H. Brune, H. Röder, K. Bromann, and K. Kern, Thin Solid Films 264, 230 (1995).

${ }^{14}$ G. Rangelov, T. Fauster, U. Strüber, and J. Küppers, Surf. Sci. 331-333, 948 (1995).

${ }^{15}$ S. C. Wang and G. Ehrlich, Phys. Rev. Lett. 67, 2509 (1991).

${ }^{16}$ H. Röder, H. Brune, J.-P. Bucher, and K. Kern, Surf. Sci. 298, 121 (1993).

${ }^{17}$ H. Brune, H. Röder, C. Boragno, and K. Kern, Phys. Rev. B 49, 2997 (1994).

${ }^{18}$ P. Zeppenfeld, M. A. Krzyzowski, C. Romainczyk, R. David, G. Cosma, H. Röder, K. Bromann, H. Brune, and K. Kern, Surf. Sci. Lett. 342, L1131 (1995).

${ }^{19}$ J. Fassbender, U. May, B. Schirmer, R. M. Jungblut, B. Hillebrands, and G. Güntherodt, Phys. Rev. Lett. 75, 4476 (1995). 